\title{
Comparative study of Biodiesel Production from Ethanol and Babassu oil using Mechanical Agitation and Ultrasounds
}

\author{
Eduardo J. Mendes de Paiva, Jayne Carlos S. Barboza, Maria Lucia Caetano Pinto da Silva, \\ Heizir Ferreira de Castro and Domingos Sávio Giordani \\ Department of Chemical Engineering \\ School of Engineering of Lorena - University of São Paulo \\ CP 116 - 12600-970 - Lorena (Brazil) \\ Phone/Fax number:+00551231533224, e-mail: giordani@dequi.eel.usp.br
}

\begin{abstract}
Babassu oil is a vegetable oil extracted from the seeds of the babassu palm (Attalea speciosa), which grows in most areas of South America. Brazil is the world's largest exporter of ethanol; the advantages of this alcohol are concerned to its renewable origin and low toxicity. In this work ethyl esters of babassu oil were synthesized by alkaline catalysis in homogeneous medium. The experimental design was used as a tool for optimization of the transesterification reaction and also in identifying key factors influencing the conversion into ethyl esters. The transesterification reactions were performed using two methods - the traditional mechanical agitation and agitation promoted by ultrasound waves. The nuclear magnetic resonance spectroscopy was used to quantify the conversion of all reactions of transesterification. According to the model obtained for mechanical agitation, conversions above $99 \%$ are obtained when the stoichiometric ratio is set at $6: 1$, with $1.0 \% \mathrm{KOH}$, under stirring at $400 \mathrm{rpm}$, in 60 minutes. Alkaline transesterification assisted by ultrasound waves produced the best results with respect to time of reaction and phase separation of glycerin and ethyl esters. The experimental model showed that conversions above $99 \%$ can be obtained in 10 minutes after adjusting the other independent variables.
\end{abstract}

\section{Key words}

Biodiesel, Babassu oil, Transesterification, Ethanol, Ultrasound

\section{Introduction}

The transesterification of vegetable oils is still the most viable and immediate alternative to reduce the high viscosity of vegetable oils [1]. However, the reaction produces a mixture of esters, free glycerol and non reacted alcohol, catalyst, mono-, di- and triglycerides.

Achieving high conversions in mono-alkyl esters and ensure the removal of contaminants in biodiesel are the main challenges to be overcome. On the other hand, the fatty acid composition of vegetable oils is also a significant factor related to the performance of biofuels, and saturated carbon chains of vegetable oils are particularly desirable [2].

The babassu is a promising feedstock, which kernel is constituted of $66 \%$ by weight of oil of predominantly saturated composition (83\%), factors that makes of this oil an excellent candidate for biodiesel production.

In fact, in Brazil many oilseed crops can be used in biodiesel production; currently the greatest part uses soybean oil as the raw material. However, soybean oil is preferably used by the food industry and other oilseeds have being researched to produce biodiesel. In this sense, non edible crops, as the babassu oil which presents annual productivity and a good yield per hectare, have became excellent alternative.

This work aimed to study the production of biodiesel from babassu oil and ethanol. The use of this alcohol is beneficial because it results in a $100 \%$ renewable biofuel in synergy with the growing international market for ethanol. The technological route used was alkaline homogeneous catalysis. Mechanical agitation, the most accessible technology, was used and compared with the use of ultrasounds in the reaction.

\section{Material and Methods}

Refined babassu oil was kindly provided by the company COGNIS Brazil Ltd. NaOH (99\%), KOH (85\%), anhydrous ethanol (99.8\%) were used as received from supplier.

The influences of the major controllable variables of the transesterification reaction were evaluated using the experimental design, with Taguchi orthogonal arrays. The levels of controllable factors were chosen based on 
the literature $[3,4]$. Tables 1 and 2 show the variables used in studies and their respective levels.

The general procedure for transesterification was done by mixing sodium hydroxide and anhydrous ethanol at $40^{\circ} \mathrm{C}$ under magnetic stirring until complete dissolution. Then this ethoxide solution was added to $70 \mathrm{~g}$ of refined babassu oil, previously heated to the reaction temperature.

Table 1. Levels of controllable factors in the reaction using mechanical agitation

\begin{tabular}{|l|c|c|c|}
\hline \multicolumn{1}{|c|}{ Factors } & Unity & \multicolumn{2}{c|}{ Levels } \\
\hline & & 1 & 2 \\
\hline Turbulence level & $\mathrm{rpm}$ & 200 & 400 \\
\hline Temperature & ${ }^{\circ} \mathrm{C}$ & 30 & 60 \\
\hline Mol ratio ethanol/oil & & $4: 1$ & $6: 1$ \\
\hline Ratio hydroxide/oil & & 0.5 & 1.0 \\
\hline Time of reaction & $\min$ & 30 & 60 \\
\hline Hydroxide & & $\mathrm{NaOH}$ & $\mathrm{KOH}$ \\
\hline
\end{tabular}

Table 2. Levels of controllable factors in reactions using ultrasounds

\begin{tabular}{|l|c|c|c|}
\hline \multicolumn{1}{|c|}{ Factors } & Unity & \multicolumn{2}{c|}{ Levels } \\
\hline & & 1 & 2 \\
\hline Mol ratio ethanol/oil & & $3: 1$ & $6: 1$ \\
\hline Ratio hydroxide/oil & & 0.5 & 1.0 \\
\hline Time of reaction & min & 10 & 20 \\
\hline Hydroxide & & $\mathrm{NaOH}$ & $\mathrm{KOH}$ \\
\hline
\end{tabular}

Reactions were done in a jacketed glass reactor of $300 \mathrm{~mL}$ with a reflux condenser; temperature control was done by a thermostatic bath. Mechanical stirring was performed with curved double blades impeller and rotation control was done with an IKA RW20-digital model shaker.

After each reaction, the mixture produced was transfered to a separation funnel for collection of glycerin phase; 200 $\mathrm{mL}$ of hexane were added to the remaining phase containing the esters. After collection of the new glycerin phase formed, the ester was washed with a solution of $\mathrm{HCl}$ 0.1 mol. $\mathrm{L}^{-1}$ to reach $\mathrm{pH}$ around 7 . Then this phase was dried with approximately $0.5 \mathrm{~g}$ of anhydrous $\mathrm{Na}_{2} \mathrm{SO}_{4}$. After filtration, the hexane and residual ethanol were evaporated under low pressure at $72^{\circ} \mathrm{C}$.
The use of the ultrasound technique was performed using $22 \mathrm{~g}$ of refined babassu oil that were added in a erlenmeyer of $125 \mathrm{~mL}$. Concurrently, appropriate amounts of hydroxide and anhydrous ethanol were mixed until complete dissolution in a glass flask equipped with reflux condenser and moderate magnetic stirring for 20 $\min$ at $30^{\circ} \mathrm{C}$. Further, the solution containing ethoxide was added to the flask containing the oil previously heated to $30^{\circ} \mathrm{C}$. The tank of the ultrasonic bath was filled with $300 \mathrm{~mL}$ distilled water and then the flask containing the reactants was placed inside. Both the position and height of the flask were standardized with markers. The equipment was operated at power of $600 \mathrm{~W}$ and frequency of $20 \mathrm{kHz}$.

The conversion into ethyl esters was monitored by proton magnetic resonance - NMR-1 $\mathrm{H}$, via a Varian $300 \mathrm{MHz}$ spectrometer, using $\mathrm{CDCl}_{3}$ as solvent and $0.3 \%$ Tetramethylsilane as internal standard of chemical shift. The calculations involving the conversion of esters were determined using the formula proposed by Garcia [5]. This methodology basically consisted in the identification of molecules present in the region of 4.05 to $4.35 \mathrm{ppm}$ during a transesterification reaction by ${ }^{1} \mathrm{H}$ NMR

\section{Results and Discussion}

The tests were performed using orthogonal arrays of Taguchi L8 and L16, following the levels presented in Table 1 and 2. According to this methodology, the signal to noise ratio $(\mathrm{S} / \mathrm{N})$ can be, in this case, interpreted as conversion into ethyl esters in the presence of noise (uncontrollable) factors.

Figure 1 shows the effects of controllable factors in the signal to noise ratio, measured independently, i.e. without considering interactions between these factors.

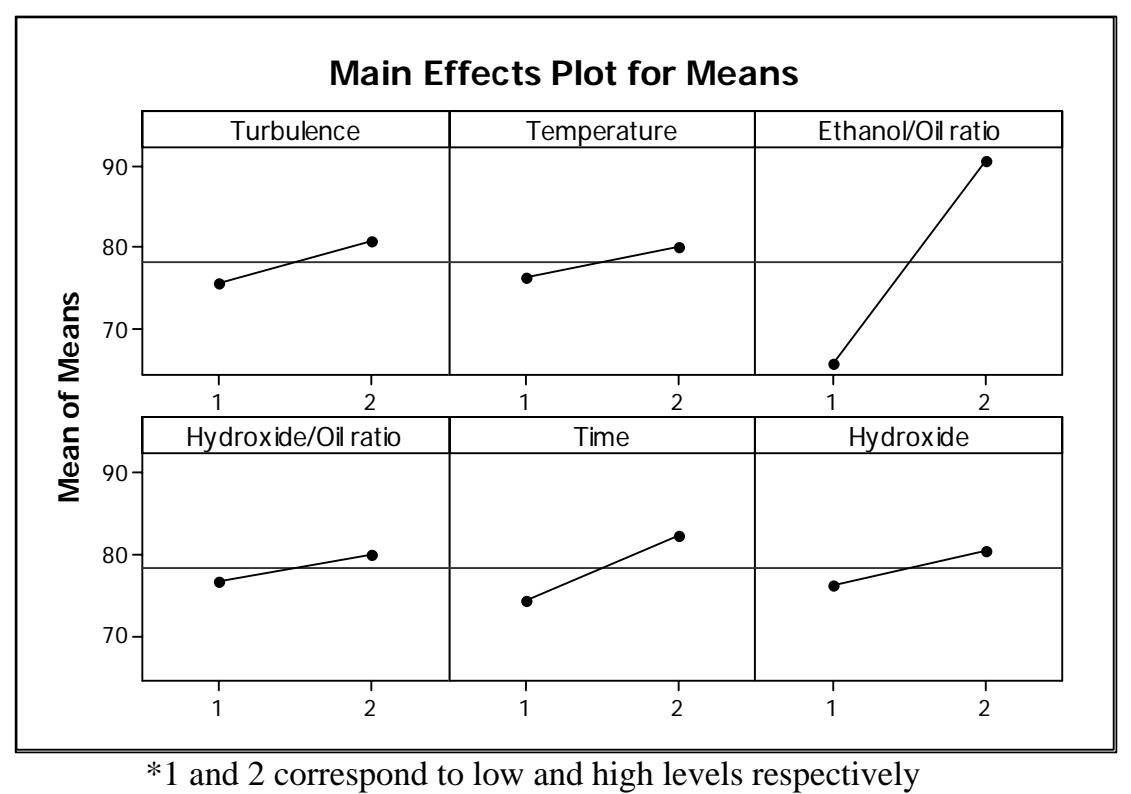

Fig. 1. Effects of controllable factors in the average conversion to ethyl esters as a function of noises 
Among all the factors evaluated, the ratio ethanol/oil was the most important factor in the conversion to ethyl esters when mechanical agitation was used. The agitation also plays an important role during the early stages of these reactions, since at this stage the mass transfer is limited $[6,7]$. The results suggest that the turbulence levels used in this study were sufficient for a good conversion, i.e., values greater than $400 \mathrm{rpm}$ do not lead to higher conversion into ethyl esters.

With regard to temperature, when evaluated independently, its effect is modest. However, considering the interactions, better conversions were obtained with a temperature of $30^{\circ} \mathrm{C}$.

Similarly, the turbulence generated by mechanical agitation at 400rpm promotes best conversion into ethyl esters with temperature of $30^{\circ} \mathrm{C}$, as it can be concluded from Figure 2. This peculiar behavior can be explained by hydrolysis and saponification reactions, enhanced by higher temperatures, which promote the consumption of the catalyst, reducing the yield of conversions.

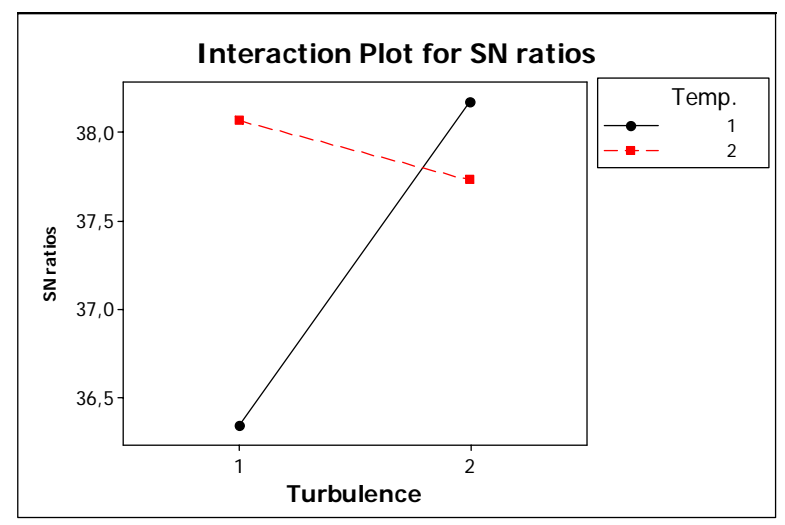

Fig. 2. Interaction of temperature/turbulence on the signal to noise ratio

The effect of the amount and the type of hydroxide used was also studied because in most industrial processes the catalyst is expensive when compared to reagents and adds additional costs for its removal of the final product.

The effective catalyst in a homogeneous transesterification reaction is the anion formed from the reaction between basic hydroxide and the alcohol. However, for industrial purposes, the percentage ratio by weight of the hydroxide to vegetable oil is commonly used to describe the effect of the catalyst involved.

Regarding the type of hydroxide, the data showed that the use of $\mathrm{KOH}$ is preferred, since smaller amounts (in mol terms) compared to the $\mathrm{NaOH}$ promoted best conversion into ethyl esters (1\% in mass of both hydroxides produce $0.0125 \mathrm{~mol}$ of potassium ethoxide against $0.0175 \mathrm{~mol}$ of sodium ethoxide), in addition, the purification procedures were significantly facilitated. Concerning the amount of hydroxide, it was shown that the use of $1 \%$ with respect to the mass of oil promotes best conversion into ethyl esters.
Finally, the reaction time showed up as a dependent factor, and without optimizing the other factors evaluated, it can be stated that satisfactory conversions were achieved with 60 minutes of reaction.

The technological model obtained was adjusted to the temperature at $30^{\circ} \mathrm{C}, 400 \mathrm{rpm}$ of mechanical agitation, using $1 \%$ potassium hydroxide during 60 minutes of reaction. The conversion into ethyl esters obtained were above $99 \%$ and the weight of esters recovered after the purification procedures was $94.59 \%$ compared to the initial mass of this product.

The evaluation of controllable factors to babassu oil ethanolysis reaction as a function of the noises was useful in determining the influence of the main variables in the conversion to ethyl esters. After that, new experimental design was employed with the two main variables, as shown in Table 2. The response surface methodology (RSM) was applied, with the development of new $2^{2}$ complete factorial design with axial points, in order to identify the optimum conditions of babassu oil ethanolysis.

The plot of Figure 3 was generated from the model obtained and show the best fit to a babassu oil ethanolysis. As can be seen, with temperatures below $40^{\circ} \mathrm{C}$ there is a narrow range in which the conversion into ethyl esters is highest, this fact makes stronger the hypothesis that temperatures between 23 and $30{ }^{\circ} \mathrm{C}$ generates the best results

In the reaction using ultrasound, the ratio ethanol/oil was the most important factor involved in ethanolysis, showing that both conventional agitation and the use of ultrasounds are primarily dependent on this factor, no matter how intense are the physical conditions to which the medium is submitted. Similar results with respect to this factor were reported in literature to the ethanolysis coconut oil [8].

It was noted that, besides the ratio ethanol/oil, the ratio hydroxide/oil is also an important factor to the reaction, which suggests a relationship between the mechanism of the reaction and cavitation effect promoted by ultrasound. In short, the best conversions with the method employing ultrasound were reached in the stoichiometric ratio of $6: 1$, with $1 \% \mathrm{KOH}$ for 10 minutes. The conversion achieved with this method was of $99.29 \%$.

The considerable time savings obtained with the ultrasound technique can be attributed to the intense reaction conditions provided by ultrasonic cavitation, which causes the reagents to react quick and efficiently, thus avoiding the formation of chemical intermediates and side reactions of hydrolysis and formation of soaps. 


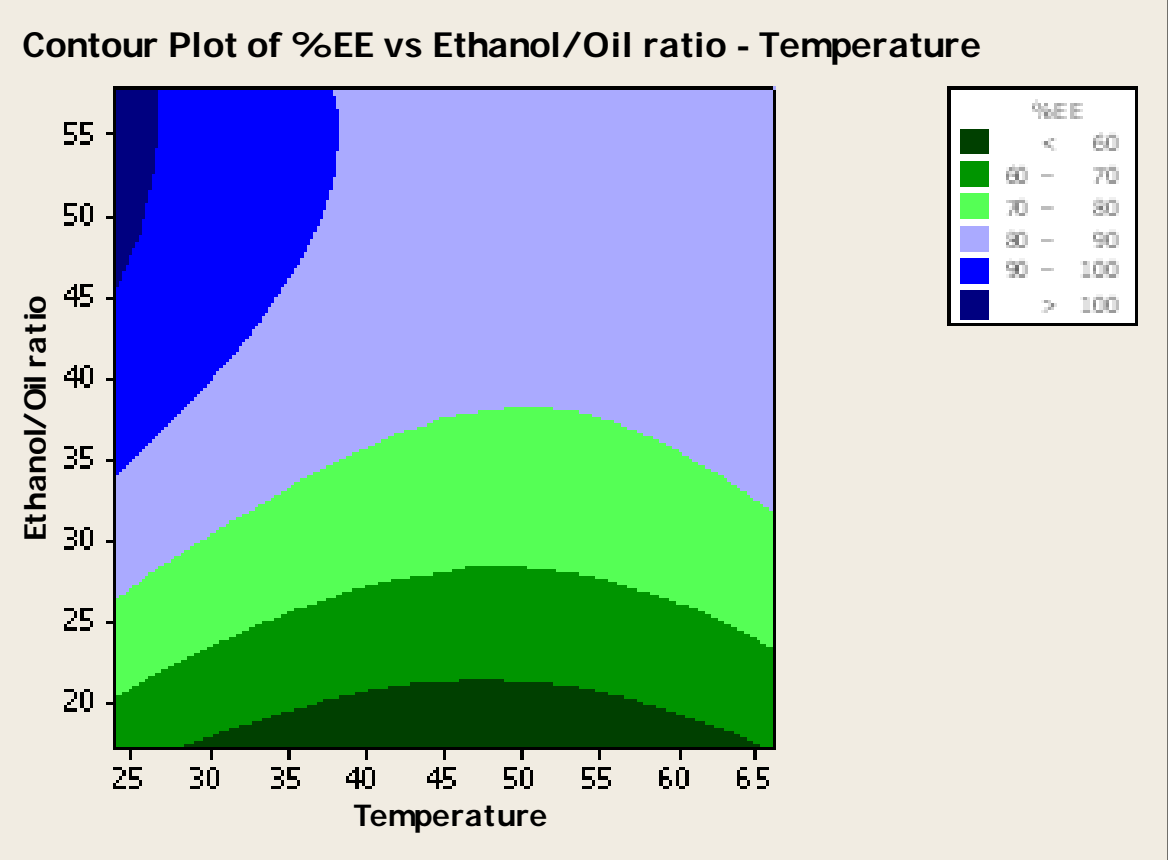

Fig. 3. Ethyl esters conversions contour plot as a function of the ethanol / oil ratio and temperature.

\section{Conclusions}

The refined babassu oil has shown excellent qualities as a raw material. Even in ethanolysis reaction this oil presented similar behavior of the reactions that used methanol showed in the literature. The experiments showed that the correct tuning in the process variables can promote higher conversions into ethyl esters.

The ratio ethanol / oil was the most influential variable in both methods.

The best conversion and phase separation conditions were obtained with the use of $\mathrm{KOH}$.

Comparison between the two methods shows that the reaction and phase separation times have been considerably reduced with the use of ultrasound.

The remarkable results obtained with the reaction time when ultrasounds was employed can be explained by intense mass transfer afforded by the unique conditions generated by cavitation noise. In addition, possible reductions in the concentrations of mono- and diglycerides during the reaction may explain the reduction in the time of phase separation.

Thus, the use of ultrasounds presents itself as a potential technological route of production of biodiesel, capable of meeting high demands in short periods of time. Adjustments related to the type of ultrasonic reactor (or transducer) and in the process of vegetable oils sonolysis can lead to an excellent alternative for biodiesel production.

\section{Acknowledgements}

Authors would like to thank to CNPq (Brazilian Council to Research), FAPESP (São Paulo State Foundation for Research) and University of São Paulo for financial Support.

\section{References}

1 Ramos, L. P., Domingos A. K., Kucek K. T., Wihelm, H. M. Rev. Biotec. Ciência \& Des. 2003, 31, 28-37.

2 Dorado, M. P.; Ballesteros, E.; Lópes, F. J.; Mittelbach, M. Energy \& Fuels, 2004, 18.

3 Fillières, R.; Benjelloun-Mlayah, B.; Delmas, M. J. Am. Oil Chem. Soc. 1995, 72.

4 Stamenkovic, O. S.; Lazic, M. L.; Todorovic, Z. B.; Veljkovic, V. B.; Skala, D. U. Bioresource Technology, 2007, 98.

5 Garcia, C. M., Master Dissertation - State University of Campinas, UNICAMP, Brazil, 2006.

6 Noureddini, H.; Zhu, D. Kinetics of transesterification of soybean oil. J. Am. Oil Chem. Soc., 1997, 74.

7 Freedman, B.; Butterfield, R. O.; Pryde, E. H. Transesterification kinetics of soybean oil, J. Am. Oil Chem. Soc., 1986, 63.

8 Kumar, D., Kumar, G., Singh, P. C. P., Fast easy ethanolysis of coconut oil for biodiesel production assisted by ultrasonication, Ultrasonics Sonochemistry, 2010, 17. 